\title{
Neutral operator with variable parameter and third-order neutral differential equation
}

\author{
Yun Xin $^{1 *}$ and Zhibo Cheng ${ }^{2}$
}

\author{
"Correspondence: \\ xy_1982@126.com \\ ${ }^{1}$ College of Computer Science and \\ Technology, Henan Polytechnic \\ University, Jiaozuo, 454000, China \\ Full list of author information is \\ available at the end of the article
}

\begin{abstract}
In this article, we discuss the properties of the neutral operator with variable parameter $(A x)(t)=x(t)-c(t) x(t-\delta(t))$ and by applying Green's function of a third-order differential equation and a fixed point theorem in cones, we obtain some sufficient conditions for existence, nonexistence, multiplicity of positive periodic solutions for a generalized third-order neutral differential equation.
\end{abstract}

Keywords: neutral operator; variable parameter; positive solutions; third-order; Green's function

\section{Introduction}

In [1], Zhang discussed the properties of the neutral operator $\left(A_{1} x\right)(t)=x(t)-c x(t-\delta)$, which became an effective tool for the research on differential equations with this prescribed neutral operator (see, e.g., [2-4]). Lu and Ge [5] investigated an extension of $A_{1}$, namely the neutral operator $\left(A_{2} x\right)(t)=x(t)-\sum_{i=1}^{n} c_{i} x\left(t-\delta_{i}\right)$, and obtained the existence of periodic solutions for the corresponding neutral differential equation. Afterwards, $\mathrm{Du}$ et al. [6] studied the neutral operator $\left(A_{3} x\right)(t)=x(t)-c(t) x(t-\delta)$, here $c(t)$ is $\omega$-periodic functions. By means of Mawhin's continuation theorem and the properties of $A_{3}$, they obtained sufficient conditions for the existence of periodic solutions to a Liénard neutral differential equation. Recently, in [7], Ren et al. investigated the neutral operator with variable delay $\left(A_{4}\right) x(t)-c x(t-\delta(t))$. By applying coincidence degree theory, they obtained sufficient conditions for the existence of periodic solutions to a Rayleigh neutral differential equation.

Motivated by [1,5-7], in this paper, we consider the neutral operator $(A x)(t)=x(t)-$ $c(t) x(t-\delta(t))$, here $|c(t)| \neq 1, c, \delta \in C^{1}(\mathbb{R}, \mathbb{R})$ and $\delta$ is an $\omega$-periodic function for some $\omega>0$. Notice that here the neutral operator $A$ is a natural generalization of the familiar operator $A_{i}, i=1,2,3,4$. But $A$ possesses a more complicated nonlinearity than $A_{i}$, $i=1,2,3,4$. For example, the neutral operator $A_{i}, i=1,2$, is homogeneous in the following sense $\left(A_{i} x\right)^{\prime}(t)=\left(A_{i} x^{\prime}\right)(t), i=1,2$, whereas the neutral operator $A$ in general is inhomogeneous. As a consequence, many of the new results for differential equations with the neutral operator $A$ will not be a direct extension of known theorems for neutral differential equations.

The paper is organized as follows. In Section 2, we first analyze qualitative properties of the generalized neutral operator $A$ which will be helpful for further studies of differential equations with this neutral operator; in Section 3, we consider a third-order neutral 
differential equation as follows:

$$
(x(t)-c(t) x(t-\delta(t)))^{\prime \prime \prime}=a(t) x(t)-\lambda b(t) f(x(t-\tau(t))),
$$

here $\lambda$ is a positive parameter; $\delta(t)$ is said to be variable delay, $c, \delta \in C^{1}(\mathbb{R}, \mathbb{R})$ and $\delta$ is an $\omega$ periodic function for some $\omega>0, f \in C(\mathbb{R},[0, \infty))$, and $f(x)>0$ for $x>0 ; a \in C(\mathbb{R},(0, \infty))$ with $\max \{a(t): t \in[0, \omega]\}<\frac{64}{81 \sqrt{3}}\left(\frac{\pi}{\omega}\right)^{3}, b \in C(\mathbb{R},(0, \infty)), \tau \in C(\mathbb{R}, \mathbb{R}), a(t), b(t)$ and $\tau(t)$ are $\omega$-periodic functions. By applying Green's function of a third-order differential equation and a fixed point theorem in cones, we obtain sufficient conditions for the existence, multiplicity and nonexistence of positive periodic solutions to the third-order neutral differential equation. We will give an example to illustrate our results, and an example is also given in this section. Our results improve and extend the results in [6-10].

\section{Analysis of the generalized neutral operator with variable parameter}

Let

$$
c_{\infty}=\max _{t \in[0, \omega]}|c(t)|, \quad c_{0}=\min _{t \in[0, \omega]}|c(t)| .
$$

Let $X=\{x \in C(\mathbb{R}, \mathbb{R}): x(t+\omega)=x(t), t \in \mathbb{R}\}$ with the norm $\|x\|=\max _{t \in[0, \omega]}|x(t)|$, and let $C_{\omega}^{+}=\{x \in C(\mathbb{R},(0, \infty)): x(t+\omega)=x(t)\}, C_{\omega}^{-}=\{x \in C(\mathbb{R},(-\infty, 0)): x(t+\omega)=x(t)\}$. Then $(X,\|\cdot\|)$ is a Banach space. A cone $K$ in $X$ is defined by $K=\{x \in X: x(t) \geq \alpha\|x\|, \forall t \in \mathbb{R}\}$, where $\alpha$ is a fixed positive number with $\alpha<1$. Moreover, define operators $A, B: C_{\omega} \rightarrow C_{\omega}$ by

$$
(A x)(t)=x(t)-c(t) x(t-\delta(t)), \quad(B x)(t)=c(t) x(t-\delta(t)) .
$$

Lemma 2.1 If $|c(t)| \neq 1$, then the operator $A$ has a continuous inverse $A^{-1}$ on $C_{\omega}$, satisfying

(1)

$$
\left(A^{-1} f\right)(t)= \begin{cases}f(t)+\sum_{j=1}^{\infty} \prod_{i=1}^{j} c\left(D_{i}\right) x\left(t-\sum_{i=1}^{j} \delta\left(D_{i}\right)\right) & \text { for }|c(t)|<1, \forall f \in C_{\omega}, \\ -\frac{f(t+\delta(t))}{c(t+\delta(t))}-\sum_{j=1}^{\infty} \frac{f\left(t+\delta(t)+\sum_{i=1}^{j} \delta\left(D_{i}^{\prime}\right)\right)}{c(t+\delta(t)) \prod_{i=1}^{j} c\left(D_{i}^{\prime}\right)} & \text { for }|c(t)|>1, \forall f \in C_{\omega} .\end{cases}
$$

(2)

$$
\left|\left(A^{-1} f\right)(t)\right| \leq \begin{cases}\frac{\|f\|}{1-c_{\infty}} & \text { for } c_{\infty}<1 \forall f \in C_{\omega} \\ \frac{\|f\|}{c_{0}-1} & \text { for } c_{0}>1 \forall f \in C_{\omega} .\end{cases}
$$

(3)

$$
\int_{0}^{\omega}\left|\left(A^{-1} f\right)(t)\right| d t \leq \begin{cases}\frac{1}{1-c_{\infty}} \int_{0}^{\omega}|f(t)| d t & \text { for } c_{\infty}<1 \forall f \in C_{\omega}, \\ \frac{1}{c_{0}-1} \int_{0}^{\omega}|f(t)| d t & \text { for } c_{0}>1 \forall f \in C_{\omega} .\end{cases}
$$

Proof Case 1: $|c(t)| \leq c_{\infty}<1$.

Let $t=D_{1}$ and $D_{j}=t-\sum_{i=1}^{j} \delta\left(D_{i}\right), j=1,2, \ldots$

$$
(B x)(t)=c(t) x(t-\delta(t))=c\left(D_{1}\right) x\left(t-\delta\left(D_{1}\right)\right)
$$




$$
\begin{aligned}
\left(B^{2} x\right)(t) & =c(t) c(t-\delta(t)) x(t-\delta(t)-\delta(t-\delta(t))) \\
& =c\left(D_{1}\right) c\left(D_{2}\right) x\left(t-\delta\left(D_{1}\right)-\delta\left(D_{2}\right)\right) \\
\left(B^{3} x\right)(t) & =c(t) c(t-\delta(t)) c(t-\delta(t)-\delta(t-\delta(t))) x\left(t-\delta\left(D_{1}\right)-\delta\left(D_{2}\right)-\delta\left(D_{3}\right)\right) \\
& =c\left(D_{1}\right) c\left(D_{2}\right) c\left(D_{3}\right) x\left(t-\sum_{i=1}^{3} \delta\left(D_{i}\right)\right) .
\end{aligned}
$$

Therefore

$$
B^{j} x(t)=\prod_{i=1}^{j} c\left(D_{i}\right) x\left(t-\sum_{i=1}^{j} \delta\left(D_{i}\right)\right)
$$

and

$$
\sum_{j=0}^{\infty}\left(B^{j} f\right)(t)=f(t)+\sum_{j=1}^{\infty} \prod_{i=1}^{j} c\left(D_{i}\right) x\left(t-\sum_{i=1}^{j} \delta\left(D_{i}\right)\right) .
$$

Since $A=I-B$, we get from $\|B\| \leq c_{\infty}<1$ that $A$ has a continuous inverse $A^{-1}: C_{\omega} \rightarrow C_{\omega}$ with

$$
A^{-1}=(I-B)^{-1}=I+\sum_{j=1}^{\infty} B^{j}=\sum_{j=0}^{\infty} B^{j},
$$

here $B^{0}=I$. Then

$$
\left(A^{-1} f(t)\right)=\sum_{j=0}^{\infty}\left[B^{j} f\right](t)=f(t)+\sum_{j=1}^{\infty} \prod_{i=1}^{j} c\left(D_{i}\right) x\left(t-\sum_{i=1}^{j} \delta\left(D_{i}\right)\right),
$$

and consequently

$$
\begin{aligned}
\left|\left(A^{-1} f\right)(t)\right| & =\left|\sum_{j=0}^{\infty}\left[B^{j} f\right](t)\right| \\
& =\left|f(t)+\sum_{j=1}^{\infty} \prod_{i=1}^{j} c\left(D_{i}\right) x\left(t-\sum_{i=1}^{j} \delta\left(D_{i}\right)\right)\right| \\
& \leq\left(1+\sum_{j=1}^{\infty} c_{\infty}^{j}\right)|f|_{\infty} \\
& \leq \frac{|f|_{\infty}}{1-c_{\infty}} .
\end{aligned}
$$

Moreover,

$$
\begin{aligned}
\int_{0}^{\omega}\left|\left(A^{-1} f\right)(t)\right| d t & =\int_{0}^{\omega}\left|\sum_{j=0}^{\infty}\left(B^{j} f\right)(t)\right| d t \leq \sum_{j=0}^{\infty} \int_{0}^{\omega}\left|\left(B^{j} f\right)(t)\right| d t \\
& =\sum_{j=0}^{\infty} \int_{0}^{\omega}\left|\prod_{i=1}^{j} c\left(D_{i}\right) x\left(t-\sum_{i=1}^{j} \delta\left(D_{i}\right)\right)\right| d t \leq \frac{1}{1-c_{\infty}} \int_{0}^{\omega}|f(t)| d t .
\end{aligned}
$$


Case 2: $|c(t)|>c_{0}>1$.

Let $D_{1}^{\prime}=t, D_{j}^{\prime}=t+\sum_{i=1}^{j} \delta\left(D_{i}^{\prime}\right), j=1,2, \ldots$ And set

$$
\begin{aligned}
& E: C_{\omega} \rightarrow C_{\omega}, \quad(E x)(t)=x(t)-\frac{1}{c(t)} x(t+\delta(t)), \\
& B_{1}: C_{\omega} \rightarrow C_{\omega}, \quad\left(B_{1} x\right)(t)=\frac{1}{c(t)} x(t+\delta(t)) .
\end{aligned}
$$

By the definition of the linear operator $B_{1}$, we have

$$
\left(B_{1}^{j} f\right)(t)=\frac{1}{\prod_{i=1}^{j} c\left(D_{i}^{\prime}\right)} f\left(t+\sum_{i=1}^{j} \delta\left(D_{i}^{\prime}\right)\right)
$$

here $D_{i}$ is defined as in Case 1. Summing over $j$ yields

$$
\sum_{j=0}^{\infty}\left(B_{1}^{j} f\right)(t)=f(t)+\sum_{j=1}^{\infty} \frac{1}{\prod_{i=1}^{j} c\left(D_{i}^{\prime}\right)} f\left(t+\sum_{i=1}^{j} \delta\left(D_{i}^{\prime}\right)\right) .
$$

Since $\left\|B_{1}\right\|<1$, we obtain that the operator $E$ has a bounded inverse $E^{-1}$,

$$
E^{-1}: C_{\omega} \rightarrow C_{\omega}, \quad E^{-1}=\left(I-B_{1}\right)^{-1}=I+\sum_{j=1}^{\infty} B_{1}^{j},
$$

and $\forall f \in C_{\omega}$ we get

$$
\left(E^{-1} f\right)(t)=f(t)+\sum_{j=1}^{\infty}\left(B_{1}^{j} f\right)(t)
$$

On the other hand, from $(A x)(t)=x(t)-c(t) x(t-\delta(t))$, we have

$$
(A x)(t)=x(t)-c(t) x(t-\delta(t))=-c(t)\left[x(t-\delta(t))-\frac{1}{c(t)} x(t)\right]
$$

i.e.,

$$
(A x)(t)=-c(t)(E x)(t-\delta(t)) .
$$

Let $f \in C_{\omega}$ be arbitrary. We are looking for $x$ such that

$$
(A x)(t)=f(t)
$$

i.e.,

$$
-c(t)(E x)(t-\delta(t))=f(t)
$$

Therefore

$$
(E x)(t)=-\frac{f(t+\delta(t))}{c(t+\delta(t))}=: f_{1}(t)
$$


and hence

$$
x(t)=\left(E^{-1} f_{1}\right)(t)=f_{1}(t)+\sum_{j=1}^{\infty}\left(B_{1}^{j} f_{1}\right)(t)=-\frac{f(t+\delta(t))}{c(t+\delta(t))}-\sum_{j=1}^{\infty} B_{1}^{j} \frac{f(t+\delta(t))}{c(t+\delta(t))},
$$

proving that $A^{-1}$ exists and satisfies

$$
\begin{aligned}
{\left[A^{-1} f\right](t) } & =-\frac{f(t+\delta(t))}{c(t+\delta(t))}-\sum_{j=1}^{\infty} B_{1}^{j} \frac{f(t+\delta(t))}{c(t+\delta(t))} \\
& =-\frac{f(t+\delta(t))}{c(t+\delta(t))}-\sum_{j=1}^{\infty} \frac{f\left(t+\delta(t)+\sum_{i=1}^{j} \delta\left(D_{i}^{\prime}\right)\right)}{c(t+\delta(t)) \prod_{i=1}^{j} c\left(D_{i}^{\prime}\right)}
\end{aligned}
$$

and

$$
\left|\left[A^{-1} f\right](t)\right|=\left|-\frac{f(t+\delta(t))}{c(t+\delta(t))}-\sum_{j=1}^{\infty} \frac{f\left(t+\delta(t)+\sum_{i=1}^{j} \delta\left(D_{i}^{\prime}\right)\right)}{c(t+\delta(t)) \prod_{i=1}^{j} c\left(D_{i}^{\prime}\right)}\right| \leq \frac{\|f\|}{c_{0}-1} .
$$

Statements (1) and (2) are proved. From the above proof, (3) can easily be deduced.

Lemma 2.2 If $c(t)<0$ and $\sigma c_{\infty}<\alpha$ here $\sigma=\frac{1-c_{0}^{2}}{1-c_{\infty}^{2}}>1$, we have for $y \in K$ that

$$
\left(\frac{\alpha}{1-c_{0}^{2}}-\frac{c_{\infty}}{1-c_{\infty}^{2}}\right)\|y\| \leq\left(A^{-1} y\right)(t) \leq \frac{1}{1-c_{\infty}}\|y\| .
$$

Proof Since $c(t)<0$ and $|c(t)| \leq c_{\infty}<\sigma c_{\infty}<\alpha<1$, by Lemma 2.1, we have for $y \in K$ that

$$
\begin{aligned}
\left(A^{-1} y\right)(t) & =y(t)+\sum_{j=1}^{\infty} \prod_{i=1}^{j} c\left(D_{i}\right) y\left(s-\sum_{i=1}^{j} \delta\left(D_{i}\right)\right) \\
& =y(t)+\sum_{j \geq 1 \text { even }} \prod_{i=1}^{j} c\left(D_{i}\right) y\left(t-\sum_{i=1}^{j} \delta\left(D_{i}\right)\right)-\sum_{j \geq 1 \text { odd }} \prod_{i=1}^{j}\left|c\left(D_{i}\right)\right| y\left(t-\sum_{i=1}^{j} \delta\left(D_{i}\right)\right) \\
& \geq \alpha\|y\|+\alpha \sum_{j \geq 1 \text { even }} c_{0}^{j}\|y\|-\|y\| \sum_{j \geq 1 \text { odd }} c_{\infty}^{j} \\
& =\frac{\alpha}{1-c_{0}^{2}}\|y\|-\frac{c_{\infty}}{1-c_{\infty}^{2}}\|y\| \\
& =\left(\frac{\alpha}{1-c_{0}^{2}}-\frac{c_{\infty}}{1-c_{\infty}^{2}}\right)\|y\| .
\end{aligned}
$$

Lemma 2.3 If $c(t)>0$ and $c(t)<1$, then for $y \in K$ we have

$$
\frac{\alpha}{1-c_{0}}\|y\| \leq\left(A^{-1} y\right)(t) \leq \frac{1}{1-c_{\infty}}\|y\| .
$$

Proof Since $c(t)>0$ and $c(t)<1, \alpha<1$, by Lemma 2.1, we have for $y \in K$ that

$$
\left(A^{-1} y\right)(t)=y(t)+\sum_{j \geq 1} \prod_{i=1}^{j} c\left(D_{i}\right) y\left(t-\sum_{i=1}^{j} \delta\left(D_{i}\right)\right) \geq \alpha\|y\|+\alpha\|y\| \sum_{j \geq 1} c_{0}^{j}=\frac{\alpha}{1-c_{0}}\|y\| .
$$




\section{Positive periodic solutions for third-order neutral equations}

At first, we introduce the following Green's functions and properties of Green's functions, which can be found in [11].

Theorem 3.1 For $\rho>0$ and $h \in X$, the equation

$$
\left\{\begin{array}{l}
u^{\prime \prime \prime}-\rho^{3} u=h(t), \\
u(0)=u(\omega), \quad u^{\prime}(0)=u^{\prime}(\omega), \quad u^{\prime \prime}(0)=u^{\prime \prime}(\omega)
\end{array}\right.
$$

has a unique solution which is of the form

$$
u(t)=\int_{0}^{\omega} G_{1}(t, s)(-h(s)) d s,
$$

where

$$
G_{1}(t, s)=\left\{\begin{array}{l}
\frac{2 \exp \left(\frac{1}{2} \rho(s-t)\right)\left[\sin \left(\frac{\sqrt{3}}{2} \rho(t-s)+\frac{\pi}{6}\right)-\exp \left(-\frac{1}{2} \rho \omega\right) \sin \left(\frac{\sqrt{3}}{2} \rho(t-s-\omega)+\frac{\pi}{6}\right)\right]}{3 \rho^{2}\left(1+\exp (-\rho \omega)-2 \exp \left(-\frac{\rho \omega}{2}\right) \cos \left(\frac{\sqrt{3}}{2} \rho \omega\right)\right)}+\frac{\exp (\rho(t-s))}{3 \rho^{2}(\exp (\rho \omega)-1)}, \\
0 \leq s \leq t \leq \omega, \\
\frac{2 \exp \left(\frac{1}{2} \rho(s-t-\omega)\right)\left[\sin \left(\frac{\sqrt{3}}{2} \rho(t-s+\omega)+\frac{\pi}{6}\right)-\exp \left(-\frac{1}{2} \rho \omega\right) \sin \left(\frac{\sqrt{3}}{2} \rho(t-s)+\frac{\pi}{6}\right)\right]}{3 \rho^{2}\left(1+\exp (-\rho \omega)-2 \exp \left(-\frac{\rho \omega}{2}\right) \cos \left(\frac{\sqrt{3}}{2} \rho \omega\right)\right)}+\frac{\exp (\rho(t+\omega-s))}{3 \rho^{2}(\exp (\rho \omega)-1)}, \\
0 \leq t \leq s \leq \omega .
\end{array}\right.
$$

Theorem 3.2 For $\rho>0$ and $h \in X$, the equation

$$
\left\{\begin{array}{l}
u^{\prime \prime \prime}+\rho^{3} u=h(t), \\
u(0)=u(\omega), \quad u^{\prime}(0)=u^{\prime}(\omega), \quad u^{\prime \prime}(0)=u^{\prime \prime}(\omega)
\end{array}\right.
$$

has a unique $\omega$-periodic solution

$$
u(t)=\int_{0}^{\omega} G_{2}(t, s) h(s) d s,
$$

where

$$
G_{2}(t, s)=\left\{\begin{array}{l}
\frac{2 \exp \left(\frac{1}{2} \rho(t-s)\right)\left[\sin \left(\frac{\sqrt{3}}{2} \rho(t-s)-\frac{\pi}{6}\right)-\exp \left(\frac{1}{2} \rho \omega\right) \sin \left(\frac{\sqrt{3}}{2} \rho(t-s-\omega)-\frac{\pi}{6}\right)\right]}{3 \rho^{2}\left(1+\exp (\rho \omega)-2 \exp \left(\frac{1}{2} \rho \omega\right) \cos \left(\frac{\sqrt{3}}{2} \rho \omega\right)\right)}+\frac{\exp (\rho(s-t))}{3 \rho^{2}(1-\exp (-\rho \omega))}, \\
0 \leq s \leq t \leq \omega, \\
\frac{2 \exp \left(\frac{1}{2} \rho(t+\omega-s)\right)\left[\sin \left(\frac{\sqrt{3}}{2} \rho(t+\omega-s)-\frac{\pi}{6}\right)-\exp \left(\frac{1}{2} \rho \omega\right) \sin \left(\frac{\sqrt{3}}{2} \rho(t-s)-\frac{\pi}{6}\right)\right]}{3 \rho^{2}\left(1+\exp (\rho \omega)-2 \exp \left(\frac{1}{2} \rho \omega\right) \cos \left(\frac{\sqrt{3}}{2} \rho \omega\right)\right)}+\frac{\exp (\rho(s-t-\omega))}{3 \rho^{2}(1-\exp (-\rho \omega))}, \\
0 \leq t \leq s \leq \omega .
\end{array}\right.
$$

Now we present the properties of the Green's functions for (3.1), (3.4).

$$
l=\frac{1}{3 \rho^{2}(\exp (\rho \omega)-1)}, \quad L=\frac{3+2 \exp \left(-\frac{\rho \omega}{2}\right)}{3 \rho^{2}\left(1-\exp \left(-\frac{\rho \omega}{2}\right)\right)^{2}} .
$$


Theorem 3.3 $\int_{0}^{\omega} G_{1}(t, s) d s=\frac{1}{\rho^{3}}$ and if $\sqrt{3} \rho \omega<\frac{4}{3} \pi$ holds, then $0<l<G_{1}(t, s) \leq L$ for all $t \in[0, \omega]$ and $s \in[0, \omega]$.

Theorem 3.4 $\int_{0}^{\omega} G_{2}(t, s) d s=\frac{1}{\rho^{3}}$ and if $\sqrt{3} \rho \omega<\frac{4}{3} \pi$ holds, then $0<l<G_{2}(t, s) \leq L$ for all $[0, \omega]$ and $s \in[0, \omega]$.

Define the Banach space $X$ as in Section 2. Denote

$$
\begin{aligned}
& M=\max \{a(t): t \in[0, \omega]\}, \quad m=\min \{a(t): t \in[0, \omega]\}, \quad \rho^{3}=M, \\
& k=l(M+m)+\sigma L M, \quad k_{1}=\frac{k-\sqrt{k^{2}-4 \sigma L l M m}}{2 \sigma L M}, \quad \alpha=\frac{l\left[m-(M+m) c_{\infty}\right]}{L M\left(1-c_{\infty}\right)} .
\end{aligned}
$$

It is easy to see that $M, m, \beta, k, k_{1}>0$.

Now we consider (1.1). First let

$$
\bar{f}_{0}=\varlimsup_{x \rightarrow 0} \frac{f(x)}{x}, \quad \bar{f}_{\infty}=\varlimsup_{x \rightarrow \infty} \frac{f(x)}{x}, \quad f_{-0}=\varliminf_{x \rightarrow 0} \frac{f(x)}{x}, \quad f_{-\infty}=\varliminf_{x \rightarrow \infty} \frac{f(x)}{x},
$$

and denote

$$
\begin{array}{ll}
\bar{i}_{0}=\text { number of 0's in }\left(\bar{f}_{0}, \bar{f}_{\infty}\right), & \underline{i}_{0}=\text { number of 0's in }\left(f_{-0}, f_{-\infty}\right) ; \\
\bar{i}_{\infty}=\text { number of } \infty \text { 's in }\left(\bar{f}_{0}, \bar{f}_{\infty}\right), \quad \underline{i}_{\infty}=\text { number of } \infty \text { 's in }\left(f_{-0}, f_{-\infty}\right) .
\end{array}
$$

It is clear that $\bar{i}_{0}, \underline{i}_{0}, \bar{i}_{\infty}, \underline{i}_{\infty} \in\{0,1,2\}$. We will show that (1.1) has $\bar{i}_{0}$ or $\underline{i}_{\infty}$ positive $w$-periodic solutions for sufficiently large or small $\lambda$, respectively.

In what follows, we discuss (1.1) in two cases, namely the case where $c(t)<0$ and $\left.-c_{\infty}\right\rangle$ $-\min \left\{k_{1}, \frac{m}{M+m}\right\}$.

From $-c_{\infty}>-\frac{m}{M+m}$, we have $\alpha=\frac{l\left[m-(M+m) c_{\infty}\right]}{L M\left(1-c_{\infty}\right)}>\frac{l\left(m-(M+m) \cdot \frac{m}{M+m}\right)}{L M\left(1-c_{\infty}\right)}=0$. So, we get $\alpha>0$. Moreover, we consider the equation

$$
\sigma L M x^{2}-k x+l m=0 .
$$

Then the equation has a solution $x=k_{1}=\frac{k-\sqrt{k^{2}-4 \sigma L l M m}}{2 \sigma L M}$. From $c_{\infty}<k_{1}$, we can get

$$
\sigma L M c_{\infty}^{2}-k c_{\infty}+\operatorname{lm}<0
$$

So, we have

$$
\sigma L M c_{\infty}^{2}-(l(M+m)+\sigma L M) c_{\infty}+l m<0
$$

we get

$$
\sigma c_{\infty}>\frac{l\left[m-(M+m) c_{\infty}\right]}{L M\left(1-c_{\infty}\right)}=\alpha
$$

On the other hand, the case where $c>0$ and $c_{\infty}<\min \left\{\frac{m}{M+m}, \frac{L M-l m}{(L-l) M-l m}\right\}$ (note that $c_{\infty}<$ $\frac{m}{M+m}$ implies $\alpha>0 ; c_{\infty}<\frac{L M-l m}{(L-l) M-l m}$ implies $\left.\alpha<1\right)$. Obviously, we have $c_{\infty}<1$, which makes Lemma 2.1 applicable for both cases, and also Lemma 2.2 or 2.3, respectively. 
Let $K=\{x \in X: x(t) \geq \alpha\|x\|\}$ denote the cone in $X$ as defined in Section 2, where $\alpha$ is just as defined above. We also use $K_{r}=\{x \in K:\|x\|<r\}$ and $\partial K_{r}=\{x \in K:\|x\|=r\}$.

Let $y(t)=(A x)(t)$, then from Lemma 2.1 we have $x(t)=\left(A^{-1} y\right)(t)$. Hence $(1.1)$ can be transformed into

$$
y^{\prime \prime \prime}(t)-a(t)\left(A^{-1} y\right)(t)=-\lambda b(t) f\left(\left(A^{-1} y\right)(t-\tau(t))\right)
$$

which can be further rewritten as

$$
y^{\prime \prime \prime}(t)-a(t) y(t)+a(t) H(y(t))=-\lambda b(t) f\left(\left(A^{-1} y\right)(t-\tau(t))\right),
$$

where $H(y(t))=y(t)-\left(A^{-1} y\right)(t)=-c(t)\left(A^{-1} y\right)(t-\delta(t))$.

Now we discuss the two cases separately.

\subsection{Case I: $c(t)<0$ and $-c_{\infty}>-\min \left\{k_{1}, \frac{m}{M+m}\right\}$}

Now we consider

$$
y^{\prime \prime \prime}(t)-a(t) y(t)+a(t) H(y(t))=h(t), \quad h \in C_{\omega}^{-},
$$

and define the operators $T, \hat{H}: X \rightarrow X$ by

$$
(T h)(t)=\int_{t}^{t+\omega} G_{1}(t, s)(-h(s)) d s, \quad(\hat{H} y)(t)=-M+a(t) y(t)-a(t) H(y(t)) .
$$

Clearly $T, \hat{H}$ are completely continuous, $(T h)(t)>0$ for $h(t)<0$ and $\|\hat{H}\| \leq(M-m+$ $\left.M \frac{c_{\infty}}{1-c_{\infty}}\right)$. By Theorem 3.1, the solution of (3.9) can be written in the form

$$
y(t)=(T h))(t)+(T \hat{H} y)(t)
$$

In view of $c(t)<0$ and $-c_{\infty}>-\min \left\{k_{1}, \frac{m}{M+m}\right\}$, we have

$$
\|T \hat{H}\| \leq\|T\|\|\hat{H}\| \leq \frac{M-m+m c_{\infty}}{M\left(1-c_{\infty}\right)}<1
$$

where we used the fact $\int_{t}^{t+\omega} G_{1}(t, s) d s=\frac{1}{M}$. Hence

$$
y(t)=(I-T \hat{H})^{-1}(T h)(t) .
$$

Define an operator $P: X \rightarrow X$ by

$$
(P h)(t)=(I-T \hat{H})^{-1}(T h)(t) .
$$

Obviously, for any $h \in C_{\omega}^{-}$, if $\max \{a(t): t \in[0, \omega]\}<\frac{64}{81 \sqrt{3}}\left(\frac{\pi}{\omega}\right)^{3}, y(t)=(P h)(t)$ is the unique positive $\omega$-periodic solution of (3.9).

Lemma 3.1 $P$ is completely continuous and

$$
(T h)(t) \leq(P h)(t) \leq \frac{M\left(1-c_{\infty}\right)}{m-(M+m) c_{\infty}}\|T h\| \quad \text { for all } h \in C_{\omega}^{-} .
$$


Proof By the Neumann expansion of $P$, we have

$$
\begin{aligned}
P & =(I-T \hat{H})^{-1} T \\
& =\left(I+T \hat{H}+(T \hat{H})^{2}+\cdots+(T \hat{H})^{n}+\cdots\right) T \\
& =T+T \hat{H} T+(T \hat{H})^{2} T+\cdots+(T \hat{H})^{n} T+\cdots
\end{aligned}
$$

Since $T$ and $\hat{H}$ are completely continuous, so is $P$. Moreover, by (3.13), and recalling that $\|T \hat{H}\| \leq \frac{M-m+m c_{\infty}}{M\left(1-c_{\infty}\right)}<1$, we get

$$
(T h)(t) \leq(P h)(t) \leq \frac{M\left(1-c_{\infty}\right)}{m-(M+m) c_{\infty}}\|T h\| .
$$

Define an operator $Q: X \rightarrow X$ by

$$
Q y(t)=P\left(\lambda b(t) f\left(\left(A^{-1} y\right)(t-\tau(t))\right)\right) .
$$

Lemma 3.2 $Q(K) \subset K$.

Proof From the definition of $Q$, it is easy to verify that $Q y(t+\omega)=Q y(t)$. For $y \in K$, we have from Lemma 3.1 that

$$
\begin{aligned}
Q y(t) & =P\left(\lambda b(t) f\left(\left(A^{-1} y\right)(t-\tau(t))\right)\right) \\
& \geq T\left(\lambda b(t) f\left(\left(A^{-1} y\right)(t-\tau(t))\right)\right) \\
& =\lambda \int_{t}^{t+\omega} G_{1}(t, s) b(s) f\left[\left(A^{-1} y\right)(s-\tau(s))\right] d s \\
& \geq \lambda l \int_{0}^{\omega} b(s) f\left[\left(A^{-1} y\right)(s-\tau(s))\right] d s .
\end{aligned}
$$

On the other hand,

$$
\begin{aligned}
Q y(t) & =P\left(\lambda b(t) f\left(\left(A^{-1} y\right)(t-\tau(t))\right)\right) \\
& \leq \frac{M\left(1-c_{\infty}\right)}{m-(M+m) c_{\infty}}\left\|T\left(\lambda b(t) f\left(\left(A^{-1} y\right)(t-\tau(t))\right)\right)\right\| \\
& =\lambda \frac{M\left(1-c_{\infty}\right)}{m-(M+m) c_{\infty}} \max _{t \in[0, \omega]} \int_{t}^{t+\omega} G_{1}(t, s) b(s) f\left(\left(A^{-1} y\right)(s-\tau(s))\right) d s \\
& \leq \lambda \frac{M\left(1-c_{\infty}\right)}{m-(M+m) c_{\infty}} L \int_{0}^{\omega} b(s) f\left(\left(A^{-1} y\right)(s-\tau(s))\right) d s .
\end{aligned}
$$

Therefore

$$
Q y(t) \geq \frac{l\left[m-(M+m) c_{\infty}\right]}{L M\left(1-c_{\infty}\right)}\|Q y\|=\alpha\|Q y\|,
$$

i.e., $Q(K) \subset K$.

From the continuity of $P$, it is easy to verify that $Q$ is completely continuous in $X$. Comparing (3.8) to (3.9), it is obvious that the existence of periodic solutions for equation (3.8) is equivalent to the existence of fixed-points for the operator $Q$ in $X$. Recalling Lemma 3.2, 
the existence of positive periodic solutions for (3.8) is equivalent to the existence of fixed points of $Q$ in $K$. Furthermore, if $Q$ has a fixed point $y$ in $K$, it means that $\left(A^{-1} y\right)(t)$ is a positive $\omega$-periodic solution of (1.1).

Lemma 3.3 If there exists $\eta>0$ such that

$$
f\left(\left(A^{-1} y\right)(t-\tau(t))\right) \geq\left(A^{-1} y\right)(t-\tau(t)) \eta \quad \text { for } t \in[0, \omega] \text { and } y \in K,
$$

then

$$
\|Q y\| \geq \lambda \ln \left(\frac{\alpha}{1-c_{0}^{2}}-\frac{c_{\infty}}{1-c_{\infty}^{2}}\right) \int_{0}^{\omega} b(s) d s\|y\|, \quad y \in K .
$$

Proof By Lemma 2.2 and Lemma 3.1, we have for $y \in K$ that

$$
\begin{aligned}
Q y(t) & =P\left(\lambda b(t) f\left(\left(A^{-1} y\right)(t-\tau(t))\right)\right) \\
& \geq T\left(\lambda b(t) f\left(\left(A^{-1} y\right)(t-\tau(t))\right)\right) \\
& =\lambda \int_{t}^{t+\omega} G_{1}(t, s) b(s) f\left(\left(A^{-1} y\right)(s-\tau(s))\right) d s \\
& \geq \lambda \ln \int_{0}^{\omega} b(s)\left(A^{-1} y\right)(s-\tau(s)) d s \\
& \geq \lambda \ln \left(\frac{\alpha}{1-c_{0}^{2}}-\frac{c_{\infty}}{1-c_{\infty}^{2}}\right) \int_{0}^{\omega} b(s) d s\|y\| .
\end{aligned}
$$

Hence

$$
\|Q y\| \geq \lambda \ln \left(\frac{\alpha}{1-c_{0}^{2}}-\frac{c_{\infty}}{1-c_{\infty}^{2}}\right) \int_{0}^{\omega} b(s) d s\|y\|, \quad y \in K .
$$

Lemma 3.4 If there exists $\varepsilon>0$ such that

$$
f\left(\left(A^{-1} y\right)(t-\tau(t))\right) \leq\left(A^{-1} y\right)(t-\tau(t)) \varepsilon \quad \text { for } t \in[0, \omega] \text { and } y \in K,
$$

then

$$
\|Q y\| \leq \lambda \varepsilon \frac{L M \int_{0}^{\omega} b(s) d s}{m-(M+m) c_{\infty}}\|y\|, \quad y \in K .
$$

Proof By Lemma 2.2 and Lemma 3.1, we have

$$
\begin{aligned}
\|Q y(t)\| & \leq \lambda \frac{M\left(1-c_{\infty}\right)}{m-(M+m) c_{\infty}} L \int_{0}^{\omega} b(s) f\left(\left(A^{-1} y\right)(s-\tau(s))\right) d s \\
& \leq \lambda \frac{M\left(1-c_{\infty}\right)}{m-(M+m) c_{\infty}} L \varepsilon \int_{0}^{\omega} b(s)\left(A^{-1} y\right)(s-\tau(s)) d s \\
& \leq \lambda \varepsilon \frac{L M \int_{0}^{\omega} b(s) d s}{m-(M+m) c_{\infty}}\|y\| .
\end{aligned}
$$

Define

$$
F(r)=\max \left\{f(t): 0 \leq t \leq \frac{r}{1-c_{\infty}}\right\},
$$




$$
f_{1}(r)=\min \left\{f(t):\left(\frac{\alpha}{1-c_{0}^{2}}-\frac{c_{\infty}}{1-c_{\infty}^{2}}\right) r \leq t \leq \frac{r}{1-c_{\infty}}\right\}
$$

Lemma 3.5 If $y \in \partial K_{r}$, then

$$
\|Q y\| \geq \lambda l f_{1}(r) \int_{0}^{\omega} b(s) d s
$$

Proof By Lemma 2.2, we obtain $\left(\frac{\alpha}{1-c_{0}^{2}}-\frac{c_{\infty}}{1-c_{\infty}^{2}}\right) r \leq\left(A^{-1} y\right)(t-\tau(t)) \leq \frac{r}{1-c_{\infty}}$ for $y \in \partial K_{r}$, which yields $f\left(\left(A^{-1} y\right)(t-\tau(t))\right) \geq f_{1}(r)$. The lemma now follows analogous to the proof of Lemma 3.3.

Lemma 3.6 If $y \in \partial K_{r}$, then

$$
\|Q y\| \leq \lambda \frac{L M\left(1-c_{\infty}\right) F(r)}{m-(M+m) c_{\infty}} \int_{0}^{\omega} b(s) d s
$$

Proof By Lemma 2.2, we can have $0 \leq\left(A^{-1} y\right)(t-\tau(t)) \leq \frac{r}{1-c_{\infty}}$ for $y \in \partial K_{r}$, which yields $f\left(\left(A^{-1} y\right)(t-\tau(t))\right) \leq F(r)$. Similar to the proof of Lemma 3.4, we get the conclusion.

We quote the fixed point theorem which our results will be based on.

Lemma 3.7 [12] Let $X$ be a Banach space and $K$ be a cone in $X$. For $r>0$, define $K_{r}=$ $\{u \in K:\|u\|<r\}$. Assume that $T: \bar{K}_{r} \rightarrow K$ is completely continuous such that $T x \neq x$ for $x \in \partial K_{r}=\{u \in K:\|u\|=r\}$.

(i) If $\|T x\| \geq\|x\|$ for $x \in \partial K_{r}$, then $i\left(T, K_{r}, K\right)=0$;

(ii) If $\|T x\| \leq\|x\|$ for $x \in \partial K_{r}$, then $i\left(T, K_{r}, K\right)=1$.

Now we give our main results on positive periodic solutions for (1.1).

\section{Theorem 3.5}

(a) If $\bar{i}_{0}=1$ or 2 , then (1.1) has $\bar{i}_{0}$ positive $\omega$-periodic solutions for $\lambda>\frac{1}{f_{1}(1) l \int_{0}^{\omega} b(s) d s}>0$;

(b) If $\underline{i}_{\infty}=1$ or 2 , then (1.1) has $\underline{i}_{\infty}$ positive $\omega$-periodic solutions for $0<\lambda<\frac{m-(M+m) c_{\infty}}{L M\left(1-c_{\infty}\right) F(1) \int_{0}^{\infty} b(s) d s}$

(c) If $\bar{i}_{\infty}=0$ or $\underline{i}_{0}=0$, then (1.1) has no positive $\omega$-periodic solutions for sufficiently small or sufficiently large $\lambda>0$, respectively.

Proof (a) Choose $r_{1}=1$. Take $\lambda_{0}=\frac{1}{f_{1}\left(r_{1}\right) l \int_{0}^{\omega} b(s) d s}>0$, then for all $\lambda>\lambda_{0}$, we have from Lemma 3.5 that

$$
\|Q y\|>\|y\| \quad \text { for } y \in \partial K_{r_{1}} .
$$

Case 1. If $\bar{f}_{0}=0$, we can choose $0<\bar{r}_{2}<r_{1}$, so that $f(u) \leq \varepsilon u$ for $0 \leq u \leq \bar{r}_{2}$, where the constant $\varepsilon>0$ satisfies

$$
\lambda \varepsilon \frac{L M \int_{0}^{\omega} b(s) d s}{m-(M+m) c_{\infty}}<1 .
$$

Let $r_{2}=\left(1-c_{\infty}\right) \bar{r}_{2}$, we have $f\left(\left(A^{-1} y\right)(t-\tau(t))\right) \leq \varepsilon\left(A^{-1} y\right)(t-\tau(t))$ for $y \in K_{r_{2}}$. By Lemma 2.2, we have $0 \leq\left(A^{-1} y\right)(t-\tau(t)) \leq \frac{\|y\|}{1-c_{\infty}} \leq \bar{r}_{2}$ for $y \in \partial K_{r_{2}}$. In view of Lemma 3.4 and (3.16), we 
have for $y \in \partial K_{r_{2}}$ that

$$
\|Q y\| \leq \lambda \varepsilon \frac{L M \int_{0}^{\omega} b(s) d s}{m-(M+m) c_{\infty}}\|y\|<\|y\| .
$$

It follows from Lemma 3.7 and (3.15) that

$$
i\left(Q, K_{r_{2}}, K\right)=1, \quad i\left(Q, K_{r_{1}}, K\right)=0,
$$

thus $i\left(Q, K_{r_{1}} \backslash \bar{K}_{r_{2}}, K\right)=-1$ and $Q$ has a fixed point $y$ in $K_{r_{1}} \backslash \bar{K}_{r_{2}}$, which means that $\left(A^{-1} y\right)(t)$ is a positive $\omega$-positive solution of (1.1) for $\lambda>\lambda_{0}$.

Case 2. If $\bar{f}_{\infty}=0$, there exists a constant $\tilde{H}>0$ such that $f(u) \leq \varepsilon u$ for $u \geq \tilde{H}$, where the constant $\varepsilon>0$ satisfies

$$
\lambda \varepsilon \frac{L M \int_{0}^{\omega} b(s) d s}{m-(M+m) c_{\infty}}<1 .
$$

Let $r_{3}=\max \left\{2 r_{1}, \frac{\tilde{H}\left(1-c_{0}^{2}\right)\left(1-c_{\infty}^{2}\right)}{\alpha\left(1-c_{\infty}^{2}\right)-c_{\infty}\left(1-c_{0}^{2}\right)}\right\}$, we have $f\left(\left(A^{-1} y\right)(t-\tau(t))\right) \leq \varepsilon\left(A^{-1} y\right)(t-\tau(t))$ for $y \in$ $K_{r_{3}}$. By Lemma 2.2, we have $\left(A^{-1} y\right)(t-\tau(t)) \geq\left(\frac{\alpha}{1-c_{0}^{2}}-\frac{c_{\infty}}{1-c_{\infty}^{2}}\right)\|y\| \geq \tilde{H}$ for $y \in \partial K_{r_{3}}$. Thus by Lemma 3.4 and (3.17), we have for $y \in \partial K_{r_{3}}$ that

$$
\|Q y\| \leq \lambda \varepsilon \frac{L M \int_{0}^{\omega} b(s) d s}{m-(M+m) c_{\infty}}\|y\|<\|y\| .
$$

Recalling Lemma 3.7 and (3.15) that

$$
i\left(Q, K_{r_{3}}, K\right)=1, \quad i\left(Q, K_{r_{1}}, K\right)=0,
$$

then $i\left(Q, K_{r_{3}} \backslash \bar{K}_{r_{1}}, K\right)=1$ and $Q$ has a fixed point $y$ in $K_{r_{3}} \backslash \bar{K}_{r_{1}}$, which means that $\left(A^{-1} y\right)(t)$ is a positive $\omega$-positive solution of (1.1) for $\lambda>\lambda_{0}$.

Case 3. If $\bar{f}_{0}=\bar{f}_{\infty}=0$, from the above arguments, there exist $0<r_{2}<r_{1}<r_{3}$ such that $Q$ has a fixed point $y_{1}(t)$ in $K_{r_{1}} \backslash \bar{K}_{r_{2}}$ and a fixed point $y_{2}(t)$ in $K_{r_{3}} \backslash \bar{K}_{r_{1}}$. Consequently, $\left(A^{-1} y_{1}\right)(t)$ and $\left(A^{-1} y_{2}\right)(t)$ are two positive $\omega$-periodic solutions of (1.1) for $\lambda>\lambda_{0}$.

(b) Let $r_{1}=1$. Take $\lambda_{0}=\frac{m-(M+m) c_{\infty}}{L M\left(1-c_{\infty}\right) F\left(r_{1}\right) \int_{0}^{\infty} b(s) d s}>0$, then by Lemma 3.6 we know if $\lambda<\lambda_{0}$ then

$$
\|Q y\|<\|y\|, \quad y \in \partial K_{r_{1}} .
$$

Case 1. If $f_{-0}=\infty$, we can choose $0<\bar{r}_{2}<r_{1}$ so that $f(u) \geq \eta u$ for $0 \leq u \leq \bar{r}_{2}$, where the constant $\eta>0$ satisfies

$$
\lambda \ln \left(\frac{\alpha}{1-c_{0}^{2}}-\frac{c_{\infty}}{1-c_{\infty}^{2}}\right) \int_{0}^{\omega} b(s) d s>1
$$

Let $r_{2}=\left(1-c_{\infty}\right) \bar{r}_{2}$, we have $f\left(\left(A^{-1} y\right)(t-\tau(t))\right) \geq \eta\left(A^{-1} y\right)(t-\tau(t))$ for $y \in K_{r_{2}}$. By Lemma 2.2, we have $0 \leq\left(A^{-1} y\right)(t-\tau(t)) \leq \frac{\|y\|}{1-c_{\infty}} \leq \bar{r}_{2}$ for $y \in \partial K_{r_{2}}$. Thus by Lemma 3.3 and (3.19),

$$
\|Q y\| \geq \lambda \ln \left(\frac{\alpha}{1-c_{0}^{2}}-\frac{c_{\infty}}{1-c_{\infty}^{2}}\right) \int_{0}^{\omega} b(s) d s\|y\|>\|y\| .
$$


It follows from Lemma 3.7 and (3.18) that

$$
i\left(Q, K_{r_{2}}, K\right)=0, \quad i\left(Q, K_{r_{1}}, K\right)=1,
$$

which implies $i\left(Q, K_{r_{1}} \backslash \bar{K}_{r_{2}}, K\right)=1$ and $Q$ has a fixed point $y$ in $K_{r_{1}} \backslash \bar{K}_{r_{2}}$. Therefore $\left(A^{-1} y\right)(t)$ is a positive $\omega$-periodic solution of (1.1) for $0<\lambda<\lambda_{0}$.

Case 2. If $f_{-\infty}=\infty$, there exists a constant $\tilde{H}>0$ such that $f(u) \geq \eta u$ for $u \geq \tilde{H}$, where the constant $\eta>0$ satisfies

$$
\lambda \ln \left(\frac{\alpha}{1-c_{0}^{2}}-\frac{c_{\infty}}{1-c_{\infty}^{2}}\right) \int_{0}^{\omega} b(s) d s>1
$$

Let $r_{3}=\max \left\{2 r_{1}, \frac{\tilde{H}\left(1-c_{0}^{2}\right)\left(1-c_{\infty}^{2}\right)}{\alpha\left(1-c_{\infty}^{2}\right)-c_{\infty}\left(1-c_{0}^{2}\right)}\right\}$, we have $f\left(\left(A^{-1} y\right)(t-\tau(t))\right) \geq \eta\left(A^{-1} y\right)(t-\tau(t))$ for $y \in$ $K_{r_{3}}$. By Lemma 2.2, we have $\left(A^{-1} y\right)(t-\tau(t)) \geq\left(\frac{\alpha}{1-c_{0}^{2}}-\frac{c_{\infty}}{1-c_{\infty}^{2}}\right)\|y\| \geq \tilde{H}$ for $y \in \partial K_{r_{3}}$. Thus by Lemma 3.3 and (3.20), we have for $y \in \partial K_{r_{3}}$ that

$$
\|Q y\| \geq \lambda \ln \left(\frac{\alpha}{1-c_{0}^{2}}-\frac{c_{\infty}}{1-c_{\infty}^{2}}\right) \int_{0}^{\omega} b(s) d s\|y\|>\|y\| .
$$

It follows from Lemma 3.7 and (3.18) that

$$
i\left(Q, K_{r_{3}}, K\right)=0, \quad i\left(Q, K_{r_{1}}, K\right)=1,
$$

i.e., $i\left(Q, K_{r_{3}} \backslash \bar{K}_{r_{1}}, K\right)=-1$ and $Q$ has a fixed point $y$ in $K_{r_{3}} \backslash \bar{K}_{r_{1}}$. That means $\left(A^{-1} y\right)(t)$ is a positive $\omega$-periodic solution of (1.1) for $0<\lambda<\lambda_{0}$.

Case 3. If $f_{-0}=f_{-\infty}=\infty$, from the above arguments, $Q$ has a fixed point $y_{1}$ in $K_{r_{1}} \backslash \bar{K}_{r_{2}}$ and a fixed point $y_{2}$ in $K_{r_{3}} \backslash \bar{K}_{r_{1}}$. Consequently, $\left(A^{-1} y_{1}\right)(t)$ and $\left(A^{-1} y_{2}\right)(t)$ are two positive $\omega$-periodic solutions of (1.1) for $0<\lambda<\lambda_{0}$.

(c) By Lemma 2.2, if $y \in K$, then $\left(A^{-1} y\right)(t-\tau(t)) \geq\left(\frac{\alpha}{1-c_{0}^{2}}-\frac{c_{\infty}}{1-c_{\infty}^{2}}\right)\|y\|>0$ for $t \in[0, \omega]$.

Case 1. If $\underline{i}_{0}=0$, we have $f_{-0}>0$ and $f_{-\infty}>0$. Let $b_{1}=\min \left\{\frac{f(u)}{u} ; u>0\right\}>0$, then we obtain

$$
f(u) \geq b_{1} u, \quad u \in[0,+\infty) .
$$

Assume that $y(t)$ is a positive $\omega$-periodic solution of (1.1) for $\lambda>\lambda_{0}$, where $\lambda_{0}=$ $\frac{\left(1-c_{0}^{2}\right)\left(1-c_{\infty}^{2}\right)}{l b_{1}\left[\alpha\left(1-c_{\infty}^{2}\right)-c_{\infty}\left(1-c_{0}\right)^{2}\right] \int_{0}^{\omega} b(s) d s}>0$. Since $Q y(t)=y(t)$ for $t \in[0, \omega]$, then by Lemma 3.3 if $\lambda>\lambda_{0}$, we have

$$
\|y\|=\|Q y\| \geq \lambda l b_{1}\left(\frac{\alpha}{1-c_{0}^{2}}-\frac{c_{\infty}}{1-c_{\infty}^{2}}\right) \int_{0}^{\omega} b(s) d s\|y\|>\|y\|,
$$

which is a contradiction.

Case 2. If $\bar{i}_{\infty}=0$, we have $\bar{f}_{0}<\infty$ and $\bar{f}_{\infty}<\infty$. Let $b_{2}=\max \left\{\frac{f(u)}{u}: u>0\right\}>0$, then we obtain

$$
f(u) \leq b_{2} u, \quad u \in[0, \infty) .
$$


Assume that $y(t)$ is a positive $\omega$-periodic solution of $(1.1)$ for $0<\lambda<\lambda_{0}$, where $\lambda_{0}=$ $\frac{m-(M+m) c_{\infty}}{b_{2} L M \int_{0}^{\omega} b(s) d s}$. Since $Q y(t)=y(t)$ for $t \in[0, \omega]$, it follows from Lemma 3.4 that

$$
\|y\|=\|Q y\| \leq \lambda b_{2} \frac{L M \int_{0}^{\omega} b(s) d s}{m-(M+m) c_{\infty}}\|y\|<\|y\|
$$

which is a contradiction.

\section{Theorem 3.6}

(a) If there exists a constant $b_{1}>0$ such that $f(u) \geq b_{1} u$ for $u \in[0,+\infty)$, then (1.1) has no positive $\omega$-periodic solution for $\lambda>\frac{\left(1-c_{0}^{2}\right)\left(1-c_{\infty}^{2}\right)}{l b_{1}\left[\alpha\left(1-c_{\infty}^{2}\right)-c_{\infty}\left(1-c_{0}^{2}\right)\right] \int_{0}^{\omega} b(s) d s}$.

(b) If there exists a constant $b_{2}>0$ such that $f(u) \leq b_{2} u$ for $u \in[0,+\infty)$, then (1.1) has no positive $\omega$-periodic solution for $0<\lambda<\frac{m-(M+m) c_{\infty}}{b_{2} L M \int_{0}^{\omega} b(s) d s}$.

Proof From the proof of (c) in Theorem 3.5, we obtain this theorem immediately.

Theorem 3.7 Assume $\underline{i}_{0}=\bar{i}_{0}=\underline{i}_{\infty}=\bar{i}_{\infty}=0$, and that one of the following conditions holds:

(1) $\bar{f}_{0} \leq f_{-\infty}$;

(2) $f_{-0}>\overline{\bar{f}}_{\infty}^{\infty}$;

(3) $\bar{f}_{0} \leq f_{-\infty} \leq \bar{f}_{0} \leq \bar{f}_{\infty}$;

(4) $f_{-\infty} \leq f_{-0} \leq \bar{f}_{\infty} \leq \bar{f}_{0}$.

If

$$
\begin{aligned}
& \frac{\left(1-c_{0}^{2}\right)\left(1-c_{\infty}^{2}\right)}{l\left[\alpha\left(1-c_{\infty}^{2}\right)-c_{\infty}\left(1-c_{0}^{2}\right)\right] \int_{0}^{\omega} b(s) d s \max \left\{f_{-0}, \bar{f}_{0}, f_{-\infty}, \bar{f}_{\infty}\right\}} \\
& \quad<\lambda<\frac{m-(M+m) c_{\infty}}{L M \int_{0}^{\omega} b(s) d s \min \left\{f_{-0}, \bar{f}_{0}, f_{-\infty}, \bar{f}_{\infty}\right\}},
\end{aligned}
$$

then (1.1) has one positive $\omega$-periodic solution.

Proof Case 1. If $\bar{f}_{0} \leq f_{-\infty}$, then

$$
\frac{\left(1-c_{0}^{2}\right)\left(1-c_{\infty}^{2}\right)}{l\left[\alpha\left(1-c_{\infty}^{2}\right)-c_{\infty}\left(1-c_{0}^{2}\right)\right] \int_{0}^{\omega} b(s) d s}<\lambda<\frac{m-(M+m) c_{\infty}}{L M \int_{0}^{\omega} b(s) d s}
$$

It is easy to see that there exists $0<\varepsilon<f_{\infty}$ such that

$$
\frac{\left(1-c_{0}^{2}\right)\left(1-c_{\infty}^{2}\right)}{\left(\bar{f}_{\infty}-\varepsilon\right) l\left[\alpha\left(1-c_{\infty}^{2}\right)-c_{\infty}\left(1-c_{0}^{2}\right)\right] \int_{0}^{\omega} b(s) d s}<\lambda<\frac{m-(M+m) c_{\infty}}{\left(f_{-0}+\varepsilon\right) L M \int_{0}^{\omega} b(s) d s}
$$

For the above $\varepsilon$, we choose $\bar{r}_{1}>0$ such that $f(u) \leq\left(f_{0}+\varepsilon\right) u$ for $0 \leq u \leq \bar{r}_{1}$. Let $r_{1}=\left(1-c_{\infty}\right) \bar{r}_{1}$, we have $f\left(\left(A^{-1} y\right)(t-\tau(t))\right) \leq\left(f_{-0}+\varepsilon\right)\left(A^{-1} y\right)(t-\tau(t))$ for $y \in K_{r_{1}}$. By Lemma 2.2, we have $0 \leq\left(A^{-1} y\right)(t-\tau(t)) \leq \frac{\|y\|}{1-c_{\infty}} \leq \bar{r}_{1}$ for $K \in \partial K_{r_{1}}$. Thus by Lemma 3.4 we have for $y \in \partial K_{r_{1}}$ that

$$
\|Q y\| \leq \lambda\left(f_{-0}+\varepsilon\right) \frac{L M \int_{0}^{\omega} b(s) d s}{m-(M+m) c_{\infty}}\|y\|<\|y\| .
$$


On the other hand, there exists a constant $\tilde{H}>0$ such that $f(u) \geq\left(\bar{f}_{\infty}-\varepsilon\right) u$ for $u \geq \tilde{H}$. Let $r_{2}=\max \left\{2 r_{1}, \frac{\tilde{H}\left(1-c_{0}^{2}\right)\left(1-c_{\infty}^{2}\right)}{\alpha\left(1-c_{\infty}^{2}\right)-c_{\infty}\left(1-c_{0}^{2}\right)}\right\}$, we have $f\left(\left(A^{-1} y\right)(t-\tau(t))\right) \geq\left(\bar{f}_{\infty}-\varepsilon\right)\left(A^{-1} y\right)(t-\tau(t))$ for $y \in K_{r_{2}}$. By Lemma 2.2, we have $\left(A^{-1} y\right)(t-\tau(t)) \geq\left(\frac{\alpha}{1-c_{0}^{2}}-\frac{c_{\infty}}{1-c_{\infty}^{2}}\right)\|y\| \geq \tilde{H}$ for $y \in \partial K_{r_{2}}$. Thus by Lemma 3.3, for $y \in \partial K_{r_{2}}$,

$$
\|Q y\| \geq \lambda l\left(\bar{f}_{\infty}-\varepsilon\right)\left(\frac{\alpha}{1-c_{0}^{2}}-\frac{c_{\infty}}{1-c_{\infty}^{2}}\right) \int_{0}^{\omega} b(s) d s\|y\|>\|y\|
$$

It follows from Lemma 3.7 that

$$
i\left(Q, K_{r_{1}}, K\right)=1, \quad i\left(Q, K_{r_{2}}, K\right)=0,
$$

thus $i\left(Q, K_{r_{2}} \backslash \bar{K}_{r_{1}}, K\right)=-1$ and $Q$ has a fixed point $y$ in $K_{r_{2}} \backslash \bar{K}_{r_{1}}$. So $\left(A^{-1} y\right)(t)$ is a positive $\omega$-periodic solution of (1.1).

Case 2. If $f_{-0}>\bar{f}_{\infty}$, in this case, we have

$$
\frac{\left(1-c_{0}^{2}\right)\left(1-c_{\infty}^{2}\right)}{\bar{f}_{0} l\left[\alpha\left(1-c_{\infty}^{2}\right)-c_{\infty}\left(1-c_{0}^{2}\right)\right] \int_{0}^{\omega} b(s) d s}<\lambda<\frac{m-(M+m) c_{\infty}}{f_{-\infty} L M \int_{0}^{\omega} b(s) d s} .
$$

It is easy to see that there exists $0<\varepsilon<f_{0}$ such that

$$
\frac{\left(1-c_{0}^{2}\right)\left(1-c_{\infty}^{2}\right)}{\left(\bar{f}_{0}-\varepsilon\right) l\left[\alpha\left(1-c_{\infty}^{2}\right)-c_{\infty}\left(1-c_{0}^{2}\right)\right] \int_{0}^{\omega} b(s) d s}<\lambda<\frac{m-(M+m) c_{\infty}}{\left(f_{\infty}+\varepsilon\right) L M \int_{0}^{\omega} b(s) d s} .
$$

For the above $\varepsilon$, we choose $\bar{r}_{1}>0$ such that $f(u) \geq\left(\bar{f}_{0}-\varepsilon\right) u$ for $0 \leq u \leq \bar{r}_{1}$. Let $r_{1}=\left(1-c_{\infty}\right) \bar{r}_{1}$, we have $f\left(\left(A^{-1} y\right)(t-\tau(t))\right) \geq\left(\bar{f}_{0}-\varepsilon\right)\left(A^{-1} y\right)(t-\tau(t))$ for $y \in K_{r_{1}}$. By Lemma 2.2, we have $0 \leq\left(A^{-1} y\right)(t-\tau(t)) \leq \frac{\|y\|}{1-c_{\infty}} \leq \bar{r}_{1}$ for $y \in \partial K_{r_{1}}$. Thus we have by Lemma 3.3 that for $y \in \partial K_{r_{1}}$,

$$
\|Q y\| \geq \lambda l\left(\bar{f}_{0}-\varepsilon\right)\left(\frac{\alpha}{1-c_{0}^{2}}-\frac{c_{\infty}}{1-c_{\infty}^{2}}\right) \int_{0}^{\omega} b(s) d s\|y\|>\|y\| .
$$

On the other hand, there exists a constant $\tilde{H}>0$ such that $f(u) \leq\left(f_{-\infty}+\varepsilon\right) u$ for $u \geq \tilde{H}$. Let $r_{2}=\max \left\{2 r_{1}, \frac{\tilde{H}\left(1-c_{0}^{2}\right)\left(1-c_{\infty}^{2}\right)}{\alpha\left(1-c_{\infty}\right)-c_{\infty}\left(1-c_{0}^{2}\right)}\right\}$, we have $f\left(\left(A^{-1} y\right)(t-\tau(t))\right) \leq\left(f_{-\infty}+\varepsilon\right)\left(A^{-1} y\right)(t-\tau(t))$ for $y \in K_{r_{2}}$. By Lemma 2.2 we have $\left(A^{-1} y\right)(t-\tau(t)) \geq\left(\frac{\alpha}{1-c_{0}^{2}}-\frac{c_{\infty}}{1-c_{\infty}^{2}}\right)\|y\| \geq \tilde{H}$ for $y \in \partial K_{r_{2}}$. Thus by Lemma 3.4, for $y \in \partial K_{r_{2}}$,

$$
\|Q y\| \leq \lambda\left(f_{-\infty}+\varepsilon\right) \frac{L M \int_{0}^{\omega} b(s) d s}{m-(M+m) c_{\infty}}\|y\| .
$$

It follows from Lemma 3.7 that

$$
i\left(Q, K_{r_{1}}, K\right)=0, \quad i\left(Q, K_{r_{2}}, K\right)=1 .
$$

Thus $i\left(Q, K_{r_{2}} \backslash \bar{K}_{r_{1}}, K\right)=-1$ and $Q$ has a fixed point $y$ in $K_{r_{2}} \backslash \bar{K}_{r_{1}}$, proving that $\left(A^{-1} y\right)(t)$ is a positive $\omega$-periodic solution of (1.1).

Case 3. $f_{0} \leq f_{\infty} \leq \bar{f}_{0} \leq \bar{f}_{\infty}$. The proof is the same as in Case 1 .

Case 4. $\bar{f}_{-\infty} \leq f_{-0} \leq \bar{f}_{\infty} \leq \bar{f}_{0}$. The proof is the same as in Case 2 . 


\subsection{Case II: $c(t)>0$ and $c_{\infty}<\min \left\{\frac{m}{M+m}, \frac{L M-I m}{(L-I) M-I m}\right\}$}

Define

$$
f_{2}(r)=\min \left\{f(t): \frac{\alpha}{1-c_{0}} r \leq t \leq \frac{r}{1-c_{\infty}}\right\}
$$

Similarly as in Section 3.1, we get the following results.

\section{Theorem 3.8}

(a) If $\bar{i}_{0}=1$ or 2 , then (1.1) has $i_{0}$ positive $\omega$-periodic solutions for $\lambda>\frac{1}{f_{2}(1) l \int_{0}^{\omega} b(s) d s}>0$.

(b) If $\underline{i}_{\infty}=1$ or 2 , then (1.1) has $i_{\infty}$ positive $\omega$-periodic solutions for $0<\lambda<\frac{m-(M+m) c_{\infty}}{L M\left(1-c_{\infty}\right) F(1) \int_{0}^{\infty} b(s) d s}$.

(c) If $\bar{i}_{\infty}=0$ or $\underline{i}_{0}=0$, then (1.1) has no positive $\omega$-periodic solution for sufficiently small or large $\lambda>0$, respectively.

\section{Theorem 3.9}

(a) If there exists a constant $b_{1}>0$ such that $f(u) \geq b_{1} u$ for $u \in[0,+\infty)$, then (1.1) has no positive $\omega$-periodic solution for $\lambda>\frac{1-c_{0}}{\operatorname{loc}_{1} \int_{0}^{\omega} b(s) d s}$.

(b) If there exists a constant $b_{2}>0$ such that $f(u) \leq b_{2} u$ for $u \in[0,+\infty)$, then (1.1) has no positive $\omega$-periodic solution for $0<\lambda<\frac{m-(M+m) c_{\infty}}{b_{2} L M \int_{0}^{\omega} b(s) d s}$.

Theorem 3.10 Assume that $\underline{i}_{0}=\bar{i}_{0}=\underline{i}_{\infty}=\bar{i}_{\infty}=0$ hold, and that one of the following conditions holds:

(1) $\bar{f}_{0} \leq f$

(2) $f_{-0}>\bar{f}_{\infty}^{\infty}$

(3) $\underline{f}_{-0} \leq f_{-\infty} \leq \bar{f}_{0} \leq \bar{f}_{\infty}$;

(4) $f_{-\infty} \leq f_{-0} \leq \bar{f}_{\infty} \leq \bar{f}_{0}$.

If

$$
\frac{1-c_{0}}{l \alpha \int_{0}^{\omega} b(s) d s \max \left\{f_{-0}, \bar{f}_{0}, f_{-\infty}, \bar{f}_{\infty}\right\}}<\lambda<\frac{m-(M+m) c_{\infty}}{L M \int_{0}^{\omega} b(s) d s \min \left\{f_{-0}, \bar{f}_{0}, f_{-\infty}, \bar{f}_{\infty}\right\}}
$$

then (1.1) has one positive $\omega$-periodic solution.

Remark 1 In a similar way, one can consider the third-order neutral functional differential equation $(x(t)-c(t) x(t-\delta(t)))^{\prime \prime \prime}+a(t) x(t)=\lambda b(t) f(x(t-\tau(t)))$.

We illustrate our results with an example.

Example 3.1 Consider the following third-order neutral differential equation:

$$
\begin{aligned}
& \left(u(t)+\frac{1}{300}\left(1-\frac{1}{2} \sin 2 t\right) u\left(t-\cos ^{2} t\right)\right)^{\prime \prime \prime}-\frac{1}{8}\left(1-\frac{1}{2} \sin ^{2} t\right) u(t) \\
& =-\lambda(1-\cos 2 t) u^{2}(t-\tau(t)) a^{u(t-\tau(t))},
\end{aligned}
$$

where $\lambda$ and $0<a<1$ are two positive parameters, $\tau(t+\pi)=\tau(t)$.

Comparing (3.21) to (1.1), we see that $\delta(t)=\cos ^{2} t, c(t)=-\frac{1}{300}\left(1-\frac{1}{2} \sin 2 t\right), a(t)=\frac{1}{8}(1-$ $\left.\frac{1}{2} \sin ^{2} t\right), b(t)=1-\cos 2 t, \omega=\pi, f(u)=u^{2} a^{u}$. Clearly, $c_{\infty}=\frac{1}{300}, c_{0}=\frac{1}{600}, M=\frac{1}{8}, m=\frac{1}{16}$, 
and we get $\rho=\frac{1}{2}$, noticing that $\frac{\sqrt{3} \pi}{2}<\frac{4 \pi}{3}$ holds. $\bar{f}_{0}=0, \bar{f}_{\infty}=0, \bar{i}_{0}=2$. By Theorem 3.5 , we easily get the following conclusion: equation (3.21) has two positive $\pi$-periodic solutions for $\lambda>\frac{7}{40 \pi r_{1}}$, where $r_{1}=\min \left\{f(0.0016), f\left(\frac{300}{299}\right)\right\}$.

In fact, by simple computations, we have

$$
\begin{aligned}
& l=\frac{1}{3 \rho(\exp (\rho \omega)-1)}=0.175, \quad L=\frac{3+2 \exp \left(-\frac{\rho \omega}{2}\right)}{3 \rho^{2}\left(1-\exp \left(-\frac{\rho \omega}{2}\right)\right)^{2}}=17.62 \\
& k=2.235, \quad k_{1}=0.0050, \quad \alpha=0.0049, \\
& c_{\infty}=\frac{1}{300}<\min \left\{k_{1}, \frac{m}{M+m}\right\}=0.0050, \quad c_{\infty}=\frac{1}{300}<0.0049=\alpha,
\end{aligned}
$$

and

$$
\begin{aligned}
& f_{1}(1)=\min \left\{f(t): 0.0016 \approx \frac{\alpha}{1-c_{0}^{2}}-\frac{c_{\infty}}{1-c_{\infty}^{2}} \leq t \leq \frac{300}{299}\right\} \\
& \quad=\min \left\{f(0.0016), f\left(\frac{300}{299}\right)\right\}=r_{1}, \\
& \frac{1}{f_{1}(1) l \int_{0}^{\pi} b(s) d s}=\frac{7}{40 \pi r_{1}} .
\end{aligned}
$$

\section{Competing interests}

The authors declare that they have no competing interests.

\section{Authors' contributions}

$Y X$ and ZBC worked together in the derivation of the mathematical results. Both authors read and approved the final manuscript.

\section{Author details}

${ }^{1}$ College of Computer Science and Technology, Henan Polytechnic University, Jiaozuo, 454000, China. ${ }^{2}$ School of Mathematics and Information Science, Henan Polytechnic University, Jiaozuo, 454000, China.

\section{Acknowledgements}

YX and ZBC would like to thank the referee for invaluable comments and insightful suggestions. This work was supported by NSFC project (Nos. 11326124, 11271339) and Education Department of Henan Province project (No. 14A110002).

Received: 16 June 2014 Accepted: 13 October 2014 Published: 24 October 2014

\section{References}

1. Zhang, M: Periodic solution of linear and quasilinear neutral functional differential equations. J. Math. Anal. Appl. 189 378-392 (1995)

2. Wang, Q, Dai, BX: Three periodic solutions of nonlinear neutral functional differential equations. Nonlinear Anal., Real World Appl. 9, 977-984 (2008)

3. Wu, J, Wang, ZC: Two periodic solutions of second-order neutral functional differential equations. J. Math. Anal. Appl. 329, 677-689 (2007)

4. Wu, J, Liu, YC: Two periodic solutions of neutral difference systems depending on two parameters. J. Comput. Appl. Math. 206, 713-725 (2007)

5. Lu, SP, Ge, WG: Existence of periodic solutions for a kind of second-order neutral functional differential equation. Appl. Math. Comput. 157, 433-448 (2004)

6. Du, B, Guo, LX, Ge, WG, Lu, SP: Periodic solutions for generalized Liénard neutral equation with variable parameter. Nonlinear Anal. TMA 70, 2387-2394 (2009)

7. Ren, JL, Cheng, ZB, Siegmund, S: Neutral operator and neutral differential equation. Abstr. Appl. Anal. 2011, Article ID $969276(2011)$

8. Ardjouni, A, Djoudi, A: Existence of periodic solutions for nonlinear neutral dynamic equations with variable delay on a time scale. Commun. Nonlinear Sci. Numer. Simul. 17, 3061-3069 (2012)

9. Bacova, B, Dorociakova, B, Olach, R: Existence of positive solutions of nonlinear neutral differential equations asymptotic to zero. Rocky Mt. J. Math. 42, 1421-1430 (2012)

10. Cheung, WS, Ren, JL, Han, WW: Positive periodic solution of second-order neutral functional differential equations. Nonlinear Anal. TMA 71, 3948-3955 (2009) 
11. Ren, JL, Siegmund, S, Chen, YL: Positive periodic solutions for third-order nonlinear differential equations. Electron. J. Differ. Equ. 2011, 66 (2011)

12. Krasnoselskii, M: Positive Solution of Operator Equation. Noordhoff, Groningen (1964)

doi:10.1186/1687-1847-2014-273

Cite this article as: Xin and Cheng: Neutral operator with variable parameter and third-order neutral differential equation. Advances in Difference Equations 2014 2014:273.

Submit your manuscript to a SpringerOpen ${ }^{\circ}$ journal and benefit from:

- Convenient online submission

- Rigorous peer review

- Immediate publication on acceptance

Open access: articles freely available online

- High visibility within the field

- Retaining the copyright to your article

Submit your next manuscript at $\boldsymbol{s p r i n g e r o p e n . c o m ~}$ 